\title{
Strategien und Techniken textilen Reparierens

\author{
Eine Exploration anhand englischer Frauenkleidung des \\ ausgehenden 19. und beginnenden 20. Jahrhunderts
}

\author{
Von PATRICIA LOCKREN
}

\begin{abstract}
Überblick
Dieser Beitrag versucht die Strategien und Techniken des textilen Reparierens, des Umarbeitens und Anpassens mit einer Analyse von Kleidung englischer Unterschichtsfrauen des ausgehenden 19. und beginnenden 20. Jahrhunderts zu rekonstruieren. Ausbesserungen und Umarbeitungen an diesen Kleidern, die mehrheitlich aus zwei Museumsbeständen und einem privaten Archiv stammen, werden dokumentiert und diskutiert. Die untersuchten Objekte unterstreichen die Omnipräsenz des Reparierens und Umarbeitens während dieser Periode und sie deuten auf Strategien der Konstruktion hin, die auf eine längere Lebensdauer bzw. die Reparaturfähigkeit dieser Kleidungsstücke abzielte.
\end{abstract}

\begin{abstract}
This article aims to explore the strategies and techniques of clothing repair, alteration and adaptation among 1900s English women from lower urban strata by analysing extant everyday clothing from this period. Repairs and alterations of these garments, which mainly stem from two museum archives and a small family collection, are documented and discussed. It is argued that the examined objects show the ubiquity of repair and alteration during this period. Furthermore, they indicate strategies of construction that enhanced the longevity of these garments and facilitated repairs.
\end{abstract}

$* * *$

Der Beitrag ${ }^{1}$ basiert auf Ergebnissen aus meinem Forschungsprojekt, ${ }^{2}$ das danach fragt, wie die Kleidung von Unterschichtsfrauen des ausgehenden 19. und beginnenden 20. Jahrhunderts aussah, wie mit dieser umgegangen wurde und wie dieselbe von den Frauen wertgeschätzt wurde. Angeregt wurde das Projekt durch den Briefbestand einer britischen Familie, der die Periode 1893 bis 1914 umfasst. Diese Familie bestand aus angelernten Perlmuttknopfma-

1 Übersetzt von Georg Stöger (Salzburg).

2 Es handelt sich hierbei um mein Disserationsprojekt „Clothes to rely on: the meanings and values of early 1900s clothing for one family's women today“, das an der Nottingham Trent University durchgeführt wird. 
chern, die im „Jewellery Quarter“ in Birmingham (UK) wohnten. Nach dem Tod der Mutter wurden im Jahre 1893 vier der acht Geschwister zur Adoption in die USA geschickt, eine Schwester kam im Erwachsenenalter nach.

Im Folgenden soll nach einer spezifischen Form des Umgangs mit Kleidung in dieser Zeit gefragt werden, dem Versuch die Lebensdauer von proletarischer Frauenkleidung zu verlängern. Welche Strategien und Techniken des textilen Reparierens, des Umarbeitens und des Anpassens lassen sich rekonstruieren? Dabei soll von Kleidungsstücken, die zwischen 1890 und 1914 entstanden sind und aus zwei Museumssammlungen, dem Besitz der Nachkommen der Emigranten und eigenen Erwerbungen bestehen, ausgegangen werden. „Außerfamiliäre“ Kleidung wurde nur herangezogen, sofern es plausibel erschien, dass sie von proletarischen Frauen in dieser Zeit getragen worden sein könnte. Somit wurde „normale“, das heißt alltägliche Kleidung und nicht die ,hohe“" Mode in den Blick genommen. Wenngleich der Fokus auf Frauenkleidung aus den Unterschichten lag, fand partiell auch Mittelschichtskleidung Berücksichtigung, da diese für proletarische Frauen infolge der weitverbreiteten Praxis der Weitergabe von „bürgerlicher" Kleidung an Dienstboten und des ausgeprägten Gebrauchtkleiderhandels zugänglich war. ${ }^{3}$ Die Analyse basiert auf Kleidung aus zwei Sammlungen in den Midlands, die beide stärker auf Spenden als auf zielgerichteten Ankäufen beruhen. Sie können eine signifikante Anzahl von Beispielen für Alltagskleidung, die zwischen den 1890er Jahren und dem Beginn des Ersten Weltkrieges entstanden ist, liefern. Für diese Periode liegen bereits Forschungen zur Kleidung wohlhabenderer Schichten ${ }^{4}$ wie auch zu der unterer sozialer Schichten vor. ${ }^{5}$

\section{Zugänge - Artefakte und Methoden}

Um sich der Frage nach textilen Reparaturen annähern zu können, wurden die zur Verfügung stehenden Kleidungsstücke in ihrer Konstruktion analysiert, zudem wurden etwaige Veränderungen durch Umarbeitungen und Ausbesserungen dokumentiert. Hierbei beziehe ich mich auf den Ansatz der Grounded Theory, die von den US-amerikanischen Soziologen Barney G. Glaser und Anselm L. Strauss in den 1960er Jahren entwickelt wurde und welche Linda

3 Penelope Byrde, Nineteenth Century Fashion, London 1992; Susan Strasser, Waste and Want. A Social History of Trash, New York 1999; Manuel Charpy, The Scope and Structure of the Nineteenth-century Second-hand Trade in the Parisian Clothes Market, in: Laurence Fontaine (Hg.), Alternative Exchanges. Second-hand Circulations from the Sixteenth Century to the Present, New York u. Oxford 2008, S. 127-151; Susan Porter Benson, Household Accounts: Working-class Family Economies in the Interwar USA, Ithaca/NY 2007.

4 Etwa im Rahmen des Victoria and Albert Museum (London); vgl. Lucy Johnston, Marion Kite u. Helen Perrson, Nineteenth Century Fashion in Detail, London 2009.

5 John Styles, The Dress of the People. Everyday Fashion in Eighteenth-century England, New Haven u. London 2007; Rachel Worth, Developing a Method for the Study of the Clothing of the ,Poor'. Some Themes in the Visual Representation of Rural Working-Class Dress, 1850-1900, in: Textile History 40, 2009, S. 70-96. 
Boynton Arthur erstmals auf die Analyse historischer Kleidung anwandte. ${ }^{6}$ Sie erweiterte dabei die Theorie um ethnographische Ansätze, die es ermöglichen sollten, ,[to] understand human behaviour in relation to clothing and appearance“.? Die Forschungsarbeit konzentriert sich dabei auf die Verwendung von Artefakten und Interviews anstatt ausschließlich auf Theorie oder Experiment zu beruhen, „the theory emerges from the data“, Arthur an. Somit wurde die Entscheidung getroffen, die Thematik mittels einer Fallstudie zu Kleidung und Kleidungspraktiken einer Arbeiterfamilie $\mathrm{zu}$ bearbeiten und auf eine breitere Untersuchung von Kleidungsstücken höherer Schichten und der Haute Couture, wie sie in den Museumssammlungen - etwa des Victoria and Albert Museum in London - dominieren, zu verzichten. ${ }^{9}$ Insgesamt wurden 89 Kleidungsstücke ausgewählt, mehrheitlich befinden sich diese in den Sammlungen des Leicestershire County Council Museum (69 Stück) und des Pickford House Museum in Derby (zehn Stück). Sechs Kleidungsstücke stammen aus dem Besitz der Nachkommen der Emigranten, die restlichen vier sind private Erwerbungen, die durch die Autorin getätigt wurden. Beide Museumsbestände wurden ausgewählt, da sie sich in den britischen Midlands befinden und somit der Herkunft der Emigranten weitgehend entsprechen, zudem ist in beiden Sammlungen Alltagskleidung vorfindbar. Gerade die Kombination dieser unterschiedlichen Bestände kann Rückschlüsse auf Kleidungspraktiken der Unterschichten um 1900, besonders im Hinblick auf das Reparieren zulassen. Dennoch sollte darauf verwiesen werden, dass Alltagskleidung - vor allem aus dem Arbeitermilieu - auch für diese Zeit relativ rar ist, Museen waren traditionell eher an der Sammlung repräsentativer Kleidung interessiert. ${ }^{10}$ Insgesamt sind nur wenige Exemplare von Alltagskleidung aus der Zeit vor dem Ersten Weltkrieg überliefert, wie Janet Arnold anmerkt: „Many have been cut up and used again, while day

6 Barney G. Glaser u. Anselm L. Strauss, The Discovery of Grounded Theory. Strategies for Qualitative Research, Chicago 1967; Linda Boynton Arthur, The Applicability of Ethnography and Grounded Theory to Clothing Research, in: Sharron J. Lennon u. Leslie Davis Burns (Hg.), Social Science Aspects of Dress. New Directions, Monument 1993, S. 137-145.

7 Boynton Arthur (wie Anm. 6), S. 143.

8 Ebd., S. 140.

9 Wenngleich Ausbesserungsarbeiten auch in der Haute Couture eine gewisse Rolle zukam, vgl. Alison Gwilt, Generating Sustainable Fashion. Opportunities, Innovation and the Creative Fashion Designer, in: Elizabeth Rouse (Hg.), Fashion and Well-being? London 2009, S. 439-452.

10 Worth (wie Anm. 5); Clare Rose, Bought, Stolen, Bqueathed, Preserved: Sources for the Study of Eighteenth-Century Petticoats, in: Maria Hayward (Hg.), Textiles and Text. Reestablishing the Links between Archival and Object-based Research, London 2009, S. 114-121; Hans-Ulrich Roller, Die Löcher im volkskundlichen Museum - flickbar?, in: Flick-Werk. Reparieren und Umnutzen in der Alltagskultur. Begleitheft zur Ausstellung im Württembergischen Landesmuseum Stuttgart vom 15. Oktober bis 15. Dezember 1983, hg. v. Ludwig-Uhland-Institut für Empirische Kulturwissenschaft der Universität Tübingen und dem Württembergischen Landesmuseum Stuttgart, Stuttgart 1983, S. 9-13. 
dresses re-trimmed and worn throughout the war period would probably have been too shabby to be worth preserving".." Der Mangel an derartigen Kleidungsstücken wird durch zwei Umstände verstärkt: zum einen durch die wenigen Forschungen zu Alltagskleidung von Frauen aus unteren sozialen Schichten (besonders aus der Arbeiterschaft), zum anderen wurden in diesem Bereich bisher noch kaum artefaktbasierende Ansätze umgesetzt. ${ }^{12}$ John Styles wandte sich in seiner Studie nichtelitärer Kleidung des 18. Jahrhundert zu und Rachel Worth untersuchte Kleidung als Form ländlicher Repräsentation. Zumindest verdeutlichen beide Arbeiten die Schwierigkeiten, denen Forschende in diesem Bereich gegenüberstehen. Worth hat zudem die Relevanz der Analyse von Kleidungsstücken betont. ${ }^{13}$ Die Analyse der Artefakte muss sorgfältig und reflektiert erfolgen, schon die Auswahl ,adäquater“ (das heißt dem Untersuchungssetting entsprechender) Kleidung ist schwierig. Es muss sichergestellt werden, dass ausschließlich Kleidung herangezogen wird, die von Frauen aus dem Arbeitermilieu der Midlands um 1900 hätte getragen werden können.

Es erscheint notwendig, um Praktiken des Tragens von Kleidung, aber auch des Umarbeitens, Anpassens und Ausbesserns, rekonstruieren zu können, die Textilien einer vollständigen Analyse zu unterziehen. Erst das Wissen um die Gesamtheit des Artefakts ermöglicht uns, zu verstehen, wie Alltagskleidung möglicherweise bereits im Hinblick auf eine möglichst lange Lebensdauer hin gefertigt wurde, und auch, ob und wie sie repariert wurde. Der Analyseprozess besteht aus drei grundlegenden Elementen: Jedes Kleidungsstück wurde fotografisch dokumentiert und mit definierten Begrifflichkeiten versehen, die der museologischen Klassifikationspraxis entsprechen. ${ }^{14}$ Dann wurden die Strukturen des Kleidungsstückes als Plan gezeichnet. Im Forschungsprozess erwiesen sich diese Zeichnungen bald als sehr zentrale Elemente, da sie einen viel klareren Eindruck vermitteln konnten, wie die Kleidungsstücke angefertigt wurden und ob die Ausführung - wie auch spätere Umarbeiten und Ausbesserungen - als professionell oder amateurhaft einzuschätzen sind. Das Zeichnen macht auch Details evident, etwa der Anfertigung oder von Reparaturen, die in den Fotografien nicht gut erkennbar waren. Zudem ermöglichte die Arbeitstechnik, die Gesamtheit des Kleidungsstückes zu dokumentieren, also die Reparaturen in ihrer Räumlichkeit und Funktionalität abzubilden.

Bei der Durchführung der Kleidungsanalyse wurde deutlich, dass keines der ausgewählten Kleidungsstücke in einem ungebrauchten Zustand war und die Objekte trugen deutliche Zeichen einer Nutzung über einen längeren Zeitraum hinweg. Dabei ist eine große Bandbreite zu beobachten, die von ,professionell‘ genähten Stücken mit zahlreichen Ausbesserungen bis hin zu

11 Janet Arnold, A Handbook of Costume, London 1974, S. 2.

12 Zur theoretischen Diskussion vgl. Jules David Prown, Mind in Matter: An Introduction to Material Culture Theory and Method, in: Winterthur Portfolio 17, 1982, H. 1, S. 1-19.

13 Worth (wie Anm. 5); Styles (wie Anm. 5).

14 Vgl. Maggie Pedley, A guide to using Spectrum, Cambridge 1998 
sehr unbeholfen bearbeiteten mit wenigen Zeichen für Reparaturen oder Adaptionen und vice versa reicht. Es war nicht immer möglich festzustellen oder abzuschätzen, wer die jeweiligen Kleidungsstücke angefertigt oder erworben hat, von wie vielen Frauen diese getragen beziehungsweise verändert oder ausgebessert wurden. Das Wissen darüber hängt sehr stark von der Qualität der Daten ab, die in den Museumssammlungen festgehalten wurden, nicht zuletzt davon, ob den Museen beim Erwerb dieser Kleidungsstücke überhaupt derartige Informationen zugänglich gemacht wurden.

\section{Techniken und Strategien des Reparierens}

Die Analyse und zeichnerische Erfassung der Kleidungsstücke offenbart eine Vielzahl von „Reparaturtechniken“ und Konstruktionen, die auf eine spätere Reparaturfähigkeit abzielen. Folgende Beispiele finden sich in Kleidungsstücken dieser Periode, die der Sammlung des Leicestershire County Council Museum entstammen: ${ }^{15}$ Ein grün-graues Seidenmieder (entstanden um 1900) weist eine eingesteckte Vorderseite auf, die eine Erweiterung zulässt und einen Abnäher in einem der Ärmel (L.C 301.1959). Ein rosafarbenes Leinenmieder mit Rock (datiert auf ca. 1910-1915), dessen Fertigung wenig professionell wirkt, weist deutliche Spuren von Anpassungen durch das Hinzufügen von großen Falten auf (L.C 61.1994). Ein Kleid für eine Brautjungfer, bestehend aus einem Rock und einem davon getrennten Mieder (datiert auf ca. 1908), erfuhr zahlreiche Änderungen: Abnäher wurden von der Taille entfernt, andere später hinzugefügt (L.C 527.1977).

Bei der Analyse der Kleidungsstücke wurde bald deutlich, dass die Kleidung bewusst so konstruiert worden war, dass durch eine Verstärkung des Stoffes eine längere Lebensdauer erreicht wurde und sie zudem ,reparaturfreundlich“ wurde - also eine spätere Ausbesserung oder Umarbeitung ermöglicht wurde. Nur ein Drittel der untersuchten Röcke wies einen Bund auf, der - wie es

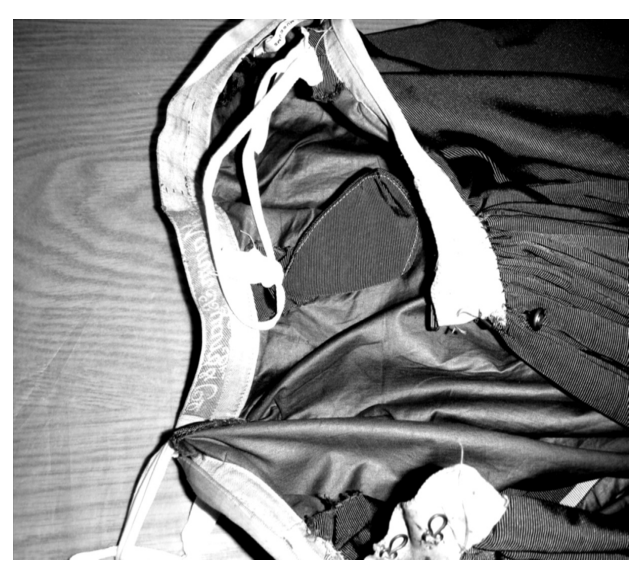
auch heute üblich ist - dem Stoff des Kleidungsstückes entsprach. Die übrigen zwei Drittel hatten Bünde, die aus einem gewebten Stoff („Petersham“) genäht waren, Abbildung 1 zeigt eine derartige Konstruktion. An diesem Rock sind zudem viele mit der Hand ausgeführte Nähte zu sehen, die zur Ausbesserung des nachgeben-

Abb. 1: Rock (um 1900) (Leicestershire County Council Museum Service, L.C 244.1963). Quelle: Fotografie der Autorin.

15 Die in Klammer angeführten Nummern bezeichnen die Signaturen der Kleidungsstücke im Leicestershire County Council Museum. 
den Stoffes beitragen sollten. Diese Konstruktionsweise zeigt, dass damalige Schneider/innen eher einen funktionalen Zugang zu der Gestaltung des Innenlebens von Kleidungsstücken hatten, zentral war offenbar eine Konstruktion, die einfachere Um- und Ausbesserungsarbeiten ermöglichen sollte.

59 Prozent der untersuchten Kleidungsstücke hatten Hauptnähte, die mit einer Nähmaschine ausgeführt wurden, dies waren jedoch - abgesehen von einer geringen Anzahl von Säumen - die einzigen maschinell ausgeführten Nähte. Möglicherweise ist dies eine Konsequenz von Defiziten der frühen Nähmaschinen: Diese waren mitunter nur begrenzt bei komplizierteren Prozessen verwendbar. Es könnte aber auch sein, dass die von Hand genähten Bereiche bereits durch Um- und Ausbesserungsarbeiten entstanden sind oder dadurch ein späteres Auftrennen der Nähte erleichtert werden sollte. Der Einsatz von Handnähten hätte zudem weniger Einstichlöcher oder andere Beschädigungen des Stoffes hinterlassen und weniger Arbeitszeit gebraucht.

In diesem Zusammenhang sollte auch die Funktion ,anpassungsfähiger“ Nähte berücksichtigt werden: Dies sind Nähte, die zur damaligen Zeit „,strategisch" ausgewählt, oftmals nicht fertig vernäht wurden und in denen der Stoff nicht beschnitten wurde, um eine Erweiterung oder Verkleinerung des Kleidungsstückes, die weder dessen Passform noch Gestalt verändern sollte, ex post zu ermöglichen. Im Falle eines Mieders wären dies die Nähte an den Schultern und unter den Achseln. Die Ausführung dieser Nähte, zeitgenössisch als „fitting seams“ bezeichnet, wird in einem britischen Schneiderhandbuch folgendermaßen beschrieben: ,tack the underarm and shoulder seam on the right side, and try on the shirt, make any necessary alterations and corrections $[\ldots]$ if the shirt is too tight across the chest, let it out at the underarm seam (another fitting seam) and draw down the front shoulder towards the armhole" ${ }^{16}$ Andere in den untersuchten Kleidungsstücken aufgefundene Reparaturtechni-

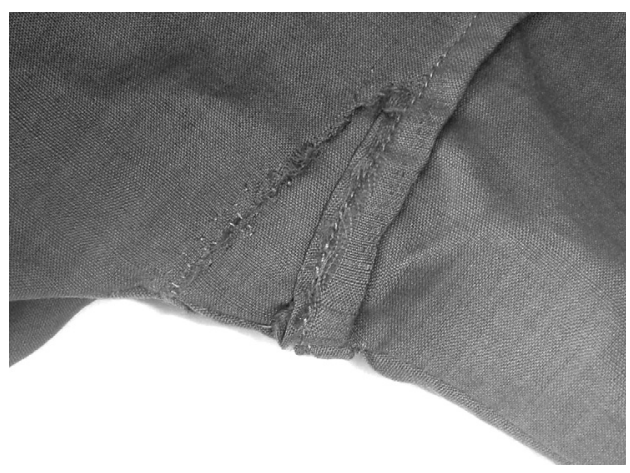

Abb. 2: Achselbereich eines Kleides (um 1914) (Leicestershire County Council Museum Service, L.C 739.1980). Quelle: Fotografie der Autorin. ken zeigen die nachträgliche Einfügung eines neuen oder auch bereits verwendeten Stoffstückes, welches eine gestopfte Stelle verstärken oder nach der Ausbesserung eines Risses die genähte Stelle entlasten sollte. Abbildung 2 zeigt ein Kleid, bei dem im Achselbereich ein dreieckiges Stück diese Zwecke erfüllte. Dieses Beispiel legt auch nahe, wie stark bereits bei der Anfertigung eines Kleidungsstückes eine mögliche spätere Reparatur im Blick behalten wurde: Die

16 M. Browne Prince, Practical Work of Dressmaking and Tailoring, London 1902, S. 18. 
Ausbesserung erfolgte mit dem gleichen Stoff, aus dem auch das übrige Kleid genäht worden war. Augenscheinlich hatte man Stoffreste beiseite gelegt. Von den untersuchten Kleidungsstücken haben insgesamt 33 Prozent größere Nähte und 42 Prozent größere Säume oder Blenden, als es gegenwärtig üblich ist - ein Beispiel hierfür ist in Abbildung 3 wiedergegeben. Hier sollte der breite Innensaum offenbar eine längere Lebensdauer bewirken.

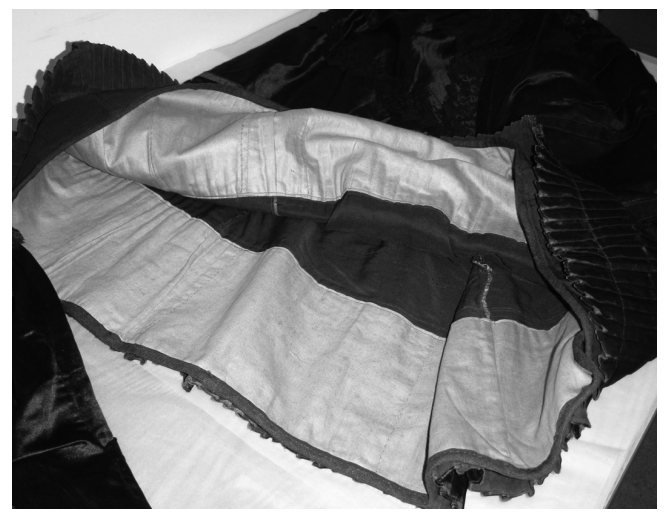

Abb. 3: Rock (um 1900) (Leicestershire County Council Museum Service, L.C 299.1959). Quelle: Fotografie der Autorin.

\section{Schlussbemerkung}

Die Relevanz des Umarbeitens und Reparierens von Alltagskleidung war noch zu Beginn des 20. Jahrhunderts für Unterschichtsangehörige hoch. Die untersuchten Kleidungsstücke weisen zu einem überwiegenden Teil Spuren von Um- und Ausbesserungsarbeiten auf (45 Prozent), zudem zielten viele Konstruktionsdetails auf eine spätere Reparaturfähigkeit ab. Kleidung war zwar zu diesem Zeitpunkt - infolge der Massenproduktion - deutlich billiger geworden, dennoch bildeten der Rückgriff auf Gebrauchtkleidung und der Versuch die Nutzungs- und Lebensdauer von Kleidung zu verlängern immer noch wichtige Haushaltsstrategien, besonders in Arbeiterhaushalten. ${ }^{17}$ Kleidung wurde noch in der ersten Hälfte des 20. Jahrhunderts häufig - inner- wie außerfamiliär - weitergegeben, dies bedingte auch die Notwendigkeit von Anpassungen und Reparaturen. Dieser Umgang mit Kleidung ging dabei vermutlich auch über einen rein ökonomischen Impetus hinaus, war also Teil einer „Recycling-Mentalität“ ${ }^{18}$ Wenn möglich, wurde Kleidung weitergenutzt und instandgesetzt. Darauf deutet auch die Konstruktion vieler Kleidungsstücke, die die Lebensdauer des äußeren Stoffes verlängern und spätere Um- und Ausbesserungsarbeiten ermöglichen sollten. So fungierten spezielle Nähte und Säume einerseits als Verstärkung des Gewandes, andererseits als „Reserve“ oder Ansatzpunkt für eine einfachere Ausbesserung oder Umarbeitung.

Gerade die Analyse von ausgewählten Kleidungsstücken ermöglicht mittels verschiedener Strategien einen Zugang zu Techniken und damit Mentalitäten des Reparierens. Hier wäre es überaus zielführend, die Datenbasis auszuweiten - in räumlicher, wie auch sozialer und zeitlicher Hinsicht -, um

17 Beate Bechthold-Comforty, Reparieren im Arbeiterhaushalt, in: Flick-Werk (wie Anm. 10), S. 101-104.

18 Vgl. dazu die Einleitung in diesem Heft. 
Unterschiede und Veränderungen in der Reparaturpraxis verfolgen zu können und damit die langsame Durchsetzung der „Wegwerf“-Kleidung nachzeichnen zu können. Das Reparieren und Anpassen von Kleidung ist gegenwärtig in der westlichen Welt kaum noch ökonomisch notwendig, es ist auch nicht beim Heranziehen von Dienstleistern - rentabel. Kleidung, zu Beginn des 20. Jahrhunderts noch als zu erhaltendes Kapital und Investition gesehen, ist zu einem kaum noch reparierten Alltagsgegenstand mit begrenzter Nutzungsdauer geworden. Vieles an diesem Wandlungsprozess ist bislang noch unklar - die Interviews, die ich mit den Nachkommen der Perlmuttknopfmacherfamilie, die heute in Nordamerika und Birmingham leben, geführt habe, geben einige Hinweise. Die Relevanz des Umarbeitens oder Ausbesserns von Kleidungsstücken scheint mit dem Alter der Frauen, ihrer finanziellen Situation und der persönlichen Einschätzung ihrer Kompetenzen im textilen Bereich zusammenzuhängen. Die Befragten erwähnten vor allem als Ausbesserungs- und Adaptionspraktiken das Kürzen von Hosen und Ärmeln oder das Austauschen eines defekten Reißverschlusses, welche aber eher als Tätigkeiten der Vergangenheit erzählt wurden. Dennoch wurde anderes, wie etwa das Annähen von Knöpfen und das Reparieren von aufgegangenen Nähten, als immer noch - durch die Frauen selbst oder durch bezahlte Dienstleister - ausgeführte Arbeiten thematisiert. Offenbar hing die Entscheidung zur Reparatur (oder Umarbeitung) stark mit dem wahrgenommenen Wert des Kleidungsstückes - entweder im Hinblick auf dessen Anschaffungspreis oder emotionale Bedeutung. Dies betont etwa die 30-jährige Amy aus Birmingham, als sie von den Reparaturen einer Hose und einer Jacke erzählt:

„I have done that. I had some work done on some trousers that I liked. They just fitted nice and the zip broke on them, so I had those, I had those repaired [...] it cost more than the trousers did, but they fitted really nice [...] I could, I know, how I could do it myself and I think I did, I have done that. [...] I just send it to a place in [...] and have that jacket, you know, the jacket sleeve shortened, yeah, I'd rather pay now, yeah. ${ }^{\text {"19 }}$

Interviews könnten - und sollten - um sich dem Wandel des Umganges mit Kleidung annähern zu können, neben der Analyse von Objekten einen wichtigen Ansatzpunkt gewähren. ${ }^{20}$

Anschrift der Verfasserin: Patricia Lockren, School of Art \& Design, Nottingham Trent University, Burton Street, Nottingham, NG1 4BU, Großbritannien. Email: N0191422@ntu.ac.uk

19 Interview mit der Autorin

20 Vgl. dazu auch die Beiträge in Flick-Werk (wie Anm. 10), S. 55-58 u. S. 69-75. 